Pesq. Vet. Bras. 35(8):762-766, agosto 2015

DOI:10.1590/S0100-736X2015000800008

\title{
Ramos colaterais do Arco aórtico do preá (Galea spixii Wagler, 1831) ${ }^{1}$
}

\author{
Radan E.M. Oliveira² ${ }^{2}$, Gleidson B. Oliveira², Procássia M.L. Barbosa², Ferdinando \\ V.F. Bezerra², José Fernando Gomes de Albuquerque ${ }^{2}$, Carlos E. Ambrósio ${ }^{3}$, \\ Maria A. Miglino e Moacir F. Oliveira $^{2 *}$
}

\begin{abstract}
Oliveira R.E.M., Oliveira G.B., Barbosa P.M.L., Bezerra F.V.F., Albuquerque J.F.G., Ambrósio C.E., Miglino M.A. \& Oliveira M.F. 2015. [Collateral branches of the aortic arch of galea (Galea spixii Wagler, 1831).] Ramos colaterais do Arco aórtico do preá (Galea spixii Wagler, 1831). Pesquisa Veterinária Brasileira 35(8):762-766. Departamento de Ciências Animais, Universidade Federal Rural do Semi-Árido, Av. Francisco Mota 572, Costa e Silva, Mossoró, RN 59.625-900, Brazil. E-mail: moacir@ufersa.edu.br

Galea spixii is a typical rodent of the caatinga belonging to the Caviidae family. Considering the lack of data on the aortic arch of the galea, this study aimed to describe the collateral branches of the aortic arch in this specie and to contributes to knowledge of its biology. Twenty males from previous studies were used which had been stored in a freezer at Multiplication Center of Wild Animals (CEMAS/UFERSA). The animals were thawed, the thoracic cavity was opened and the aorta cannulated vasculature was washed with saline and then injected with Neoprene latex colored with red pigment, yellow or white. Subsequently, the animals were fixed in formalin and after 72 hours dissected and analyzed, to obtain schematic drawings and the most representative samples were photographed. The cavy aortic arch issued as collateral branches, the brachiocephalic trunk and the left subclavian artery. The brachiocephalic trunk afforded in most of the specimens the left common carotid artery and the brachiocarotid trunk, where the right subclavian and right common carotid arteries arise. The right and left subclavian arteries in all animals studied issued the vertebral artery, internal thoracic artery, superficial cervical artery costocervical trunk and axillary artery. The pattern of formation of the aortic arch of galea was similar to that observed in other rodents, such as the kerodon, the guinea pig and chinchilla.
\end{abstract}

INDEX TERMS: Arteries, aortic arch, brachiocephalic trunk, Galea spixii. Caviidae, rodent.

RESUMO.- 0 preá é um roedor típico da caatinga pertencente à família Caviidae. Considerando a inexistência de dados sobre o arco aórtico do preá, foi realizado este estudo tendo como objetivo descrever os ramos colaterais do arco aórtico neste cavídeo, e dessa forma, contribuir com dados para biologia da espécie. Foram utilizados vinte preás ma-

\footnotetext{
${ }^{1}$ Recebido em 3 de fevereiro de 2015.

Aceito para publicação em 1 de agosto de 2015.

${ }^{2}$ Departamento de Ciências Animais, Universidade Federal Rural do Semi-Árido (Ufersa), Av. Francisco Mota 572, Costa e Silva, Mossoró, RN 59.625-900, Brasil. *Autor para correspondência: moacir@ufersa.edu.br

${ }^{3}$ Departamento de Medicina Veterinária, Faculdade de Zootecnia e Engenharia de Alimentos (FZEA), Campus da Universidade de São Paulo (USP), Av. Duque de Caxias Norte 225, Pirassununga, SP 13635-900, Brasil.

${ }^{4}$ Departamento de Cirurgia, Faculdade de Medicina Veterinária e Zootecnia (FMVZ), USP, Av. Prof. Dr. Orlando Marques de Paiva 87, Cidade Universitária, São Paulo, SP 05508-270, Brasil.
}

chos provenientes de estudos anteriores e encontravamse armazenados em freezer no Centro de Multiplicação de Animais Silvestres (CEMAS/UFERSA). Os animais foram descongelados, a cavidade torácica foi aberta, a aorta canulada e o sistema vascular lavado com solução salina e em seguida, injetado látex Neoprene corado com pigmento vermelho, amarelo ou branco. Posteriormente, os animais foram fixados em formol e depois de 72 horas, dissecados e analisados, sendo obtidos desenhos esquemáticos e os exemplares mais representativos fotografados. $\mathrm{O}$ arco aórtico do preá emitiu como ramos colaterais, o tronco braquiocefálico e a artéria subclávia esquerda. 0 tronco braquiocefálico originou na maioria das peças estudadas, a artéria carótida comum esquerda e o tronco braquiocarotídeo, do qual surgem as artérias subclávia direita e carótida comum direita. As artérias subclávias direita e esquerda em todos os animais estudados emitiram a artéria verte- 
bral, a artéria torácica interna, a artéria cervical superficial, o tronco costocervical e a artéria axilar. 0 padrão da formação do arco aórtico do preá assemelhou-se ao observado em outros roedores, tais como no mocó, no porquinho-da-índia e na chinchila.

TERMOS DE INDEXAÇÃO: Artérias, arco aórtico, tronco braquiocefálico, preá, Galea spixii, Caviidae; roedor.

\section{INTRODUÇÃO}

O preá é um roedor típico da caatinga pertencente à família Caviidae. Possui o corpo alongado, cor relativamente uniforme, sendo a superfície dorsal cinza e ventre branco, a cabeça e os olhos grandes, as orelhas curtas e arredondadas (Mendes 1987). Esta espécie tem sido criada em cativeiro, com o intuito de fornecer fonte alternativa de proteína animal, preservação da espécie e proporcionar o desenvolvimento de pesquisas (Santos et al. 2011).

Estudos acerca dos ramos colaterais do arco aórtico de mamíferos foram desenvolvidos em espécies domésticas como gatos e cães (Fernandes Filho \& Borelli 1970, Goshal 1981), ovinos (Rosa et al. 2011) e em coelhos (Kent 1983, Albuquerque et al. 1987, Souza et al. 2013). Em espécies silvestres citam-se dados de estudos em búfalos (Cortellini et al. 2000) gambá (Reckziegel et al. 2003), mão-pelada (Santos et al. 2004), lebre européia (Brudnicki et al. 2007), jaguatirica (Martins et al. 2010), veado-catingueiro (Schimming et al. 2012), tamanduá-mirim (Pinheiro et al. 2012) e em cachorro-do-mato (Engel et al. 2013). Já em roedores silvestres referenciam-se trabalhos com paca (Oliveira et al. 2001), chinchila (Araújo et al. 2004), mocó (Magalhães et al. 2007), capivara (Culau et al. 2007) e nutria (Campos et al. 2010).

Baseado nos Tratados de Anatomia dos animais domésticos, sabe-se que o arco aórtico origina vasos, em sua grande maioria, o tronco braquiocefálico, responsável por irrigar a cabeça, pescoço, membros torácicos, região cranial do tórax, bem como alguns órgãos torácicos (Nickel et al. 1981, Getty 1981, Dyce et al. 2004, König \& Liebich 2011).

Considerando que na literatura não existe informações referentes à morfologia do arco aórtico de preá, este trabalho teve como objetivo descrever os ramos colaterais do arco aórtico desta espécie, de modo a estabelecer um modelo padrão e assim contribuir com a biologia da espécie.

\section{MATERIAL E MÉTODOS}

Foram utilizados vinte preás machos, adultos, provenientes do Centro de Multiplicação de Animais Silvestre da Universidade Federal Rural do Semi-Árido (Cemas/Ufersa), licenciado junto ao IBAMA como criadouro científico (Registro no1478912). Os animais utilizados foram provenientes de estudos anteriores, autorizados pelo Comitê de Ética no Uso de Animais da Universidade Federal Rural do Semi-Árido (CEUA/Ufersa) (Processso 23091.001975/10-24) e encontravam-se armazenados em freezer.

Os animais foram descongelados e logo incisados medianamente para abertura da cavidade torácica, sendo a artéria aorta canulada, o sistema vascular lavado com solução salina aquosa a 0,9\% e em seguida, perfundido com látex Neopreme 450 (Du Pont do Brasil S.A.) corado com pigmento vermelho, amarelo ou branco
(Globo S.A. Tintas e Pigmentos). Posteriormente, os animais foram fixados em solução aquosa de formaldeído a $10 \%$ e depois de 72 horas, dissecados e analisados, sendo obtidos desenhos esquemáticos para cada sistema vascular e os exemplares mais representativos fotografados para documentação.

0 estudo baseou-se na nomenclatura adotada pelo International Committee on Veterinary Gross Anatomical Nomenclature (2005) para a denominação das estruturas identificadas e os resultados comparados com a literatura referente a estudos com roedores e outros mamíferos.

\section{RESULTADOS}

Os resultados mostram que o arco aórtico em todos os animais estudados, apresentou-se voltado para a esquerda e, em seu trajeto emitiu como ramos colaterais, o tronco braquiocefálico e a artéria subclávia esquerda, sendo estes vasos originados por tronco comum (50\%) ou como ramos independentes (50\%).

0 tronco braquiocefálico emitiu três ramos, correspondentes às artérias carótidas comum direita e esquerda e a artéria subclávia direita. Observou-se que estes ramos diferiram quanto a ordem em que surgiam do tronco braquiocefálico. Em 12 animais (60\%) o tronco braquiocefálico emitiu primeiro, a artéria carótida comum esquerda e um tronco, do qual surgem a artéria subclávia direita e a artéria carótida comum direita, o tronco braquiocarotídeo (Fig.1). Em seis (30\%), as artérias carótida comum esquerda, subclávia direita e carótida comum direita surgiram por trifurcação (Fig.2) e, em apenas dois (10\%) animais, o tronco braquiocefálico emitiu primeiramente a artéria subclávia direita e em seguida um tronco comum as artérias carótidas comum direita e esquerda, o tronco bicarotídeo (Fig.3).

As artérias subclávias direita e esquerda em 100\% dos animais emitiram cinco ramos, a artéria vertebral, a artéria torácica interna, a artéria cervical superficial, o tronco costocervical e a artéria axilar.

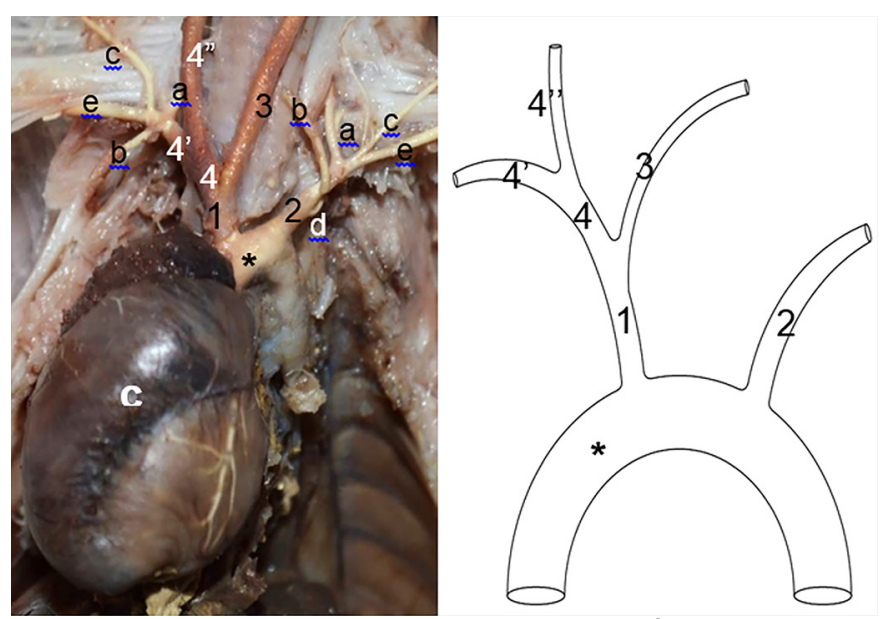

Fig.1. Arco aórtico $\left(^{*}\right)$ e seus colaterais no preá. À esquerda, verifica-se tronco braquiocefálico (1), artéria subclávia esquerda (2), carótida comum esquerda (3), tronco comum (4), subclávia direita (4') e carótida comum direita (4"), artéria vertebral (a), torácica interna (b), cervical superficial (c), tronco costocervical (d) e artéria axilar (e). À direita, desenho esquemático. 


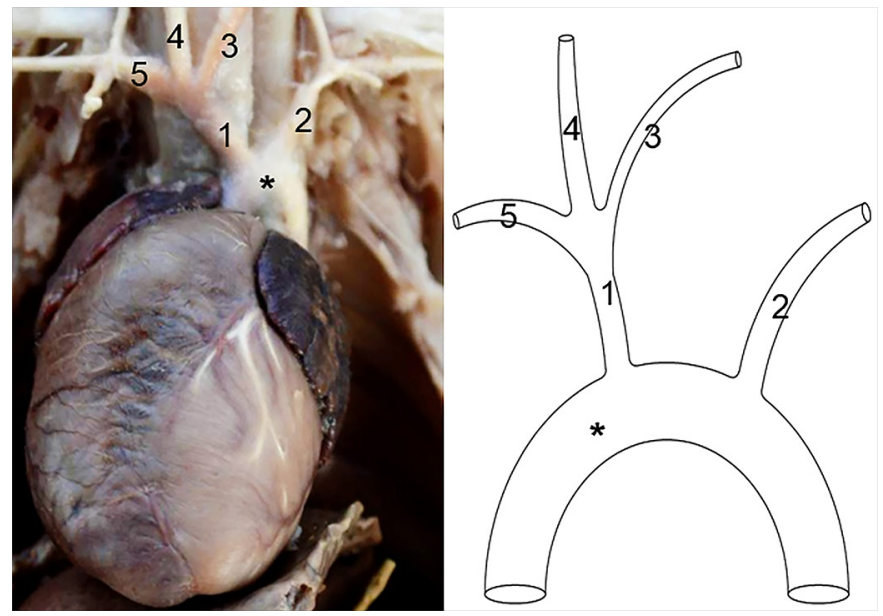

Fig.2. Arco aórtico $\left({ }^{*}\right)$ e seus colaterais no preá. À esquerda é possível observar, tronco braquiocefálico (1) e subclávia esquerda (2). Verifica-se trifurcação do tronco braquiocefálico em carótida comum esquerda (3), carótida comum direita (4) e subclávia direita (5). À direita, desenho esquemático.
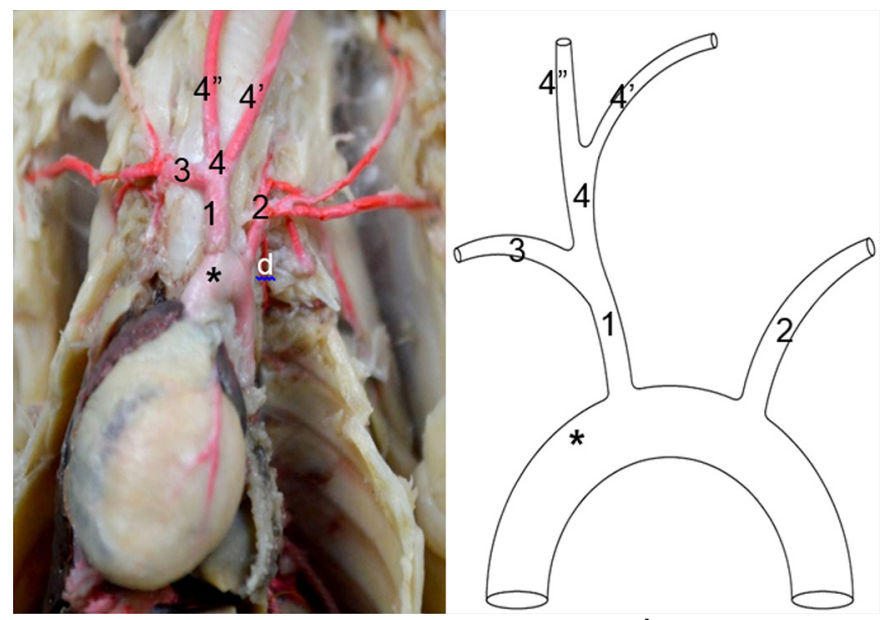

Fig.3. Arco aórtico (*) e seus colaterais no preá. À esquerda observa-se tronco braquiocefálico e subclávia esquerda (1), subclávia direita (2), subclávia direita (3), tronco bicarotídeo (4), as artérias carótida comum direita (4") e esquerda (4'), tronco costocervical (d). À direita, desenho esquemático.

\section{DISCUSSÃO}

O arco aórtico do preá emitiu como ramos colaterais, o tronco braquiocefálico e a artéria subclávia esquerda. Resultado semelhante foi encontrado por Araújo et al. (2004), estudando o arco aórtico de chinchilas, quando identificaram nesta espécie como colaterais o tronco braquiocefálico e a artéria subclávia esquerda. Estes autores relatam ainda a possibilidade do arco aórtico emitir isoladamente o tronco braquiocefálico, a artéria carótida comum esquerda e a artéria subclávia esquerda, diferente do observado em preás.

Campos et al. (2010), em experimento com arco aórtico da nutria e Souza et al. (2013) estudando os ramos colaterais do arco aórtico de coelhos, observaram em $60 \%$ e $80 \%$ dos animais analisados, respectivamente, que este origina o tronco braquiocefálico e a artéria subclávia esquerda, semelhante ao encontrado no preá.
O arco aórtico do preá teve ramificação semelhante também ao verificado em mocó (Magalhães et al. 2007) e em mamíferos como mão-pelada (Santos et al. 2004), jaguatirica (Martins et al. 2010), tamanduá-mirim (Pinheiro et al. 2012) e cachorro do mato (Engel et al. 2013). Em contrapartida, Culau et al. (2007) em trabalho realizado com capivaras, Carvalho et al. (1993) na cutia e Hebel \& Stromberg (1986) no rato de laboratório mencionam como ramo colateral do arco aórtico apenas o tronco braquiocefálico. Este padrão difere completamente do identificado para preás e outros mamíferos, assemelhando-se ao relatado no eqüino (Dyce et al. 2004, König \& Liebich 2011) e ruminantes (Dyce et al. 2004, Cortellini et al. 2000, König \& Liebich 2011, Schimming et al. 2012), como pode ser visualizado no Quadro 1.

Quanto a ordem de origem destes vasos no arco aórtico, Oliveira et al. (2001) em pacas, cita que nesta espécie o arco aórtico emite primeiramente a artéria subclávia esquerda e depois o tronco braquiocefálico. Esta ordem difere do padrão encontrado em animais domésticos e silvestres (Quadro 1) e difere do observado também em preás, cuja origem deu-se por tronco comum ou quando de forma separada, verificou-se como primeiro ramo, o tronco braquiocefálico.

0 tronco branquiocefálico no preá, após ser emitido pelo arco aórtico ramifica-se originando as artérias carótidas comum direita e em forma de um tronco, logo emite a artéria carótida esquerda, continuando seu trajeto como artéria subclávia direita. Estes resultados encontrados em preás são similares a aqueles descritos para outros roedores como pacas (Oliveira et al. 2001) e mocós (Magalhães et al. 2007), já que estes pesquisadores descrevem a presença destas artérias em $100 \%$ dos exemplares estudados.

Quanto a formação do tronco comum a artéria carótida comum direita e subclávia direita, este arranjo foi identificado no coelho (Albuquerque et al. 1987), na cutia (Carvalho et al. 1993) e na capivara (Culau et al. 2007). No entanto, estes resultados encontrados no preá diferem dos achados de Campos et al. (2010) na nutria, Souza et. al. (2013) no coelho e dos achados obtidos na chinchila por Araújo et al. (2004) quando identificaram dois padrões de formação para o tronco braquiocefálico nestes animais, sendo o primeiro formado a partir das artérias carótida comum direita e esquerda e subclávia direita e o segundo das artérias carótida comum direita e subclávia direita.

No preá, o tronco braquiocefálico originou em $30 \%$ dos casos as artérias carótidas comum direita e esquerda e a artéria subclávia direita por trifurcação, resultado também observado por Santos et al. (2004) no mão-pelada e em menor freqüência por Reckziegel et al. (2003) estudando o arco aórtico de gambás e por Magalhães et al. (2007) em mocós. Culau et al. (2007) também descreveu este mesmo comportamento em $28,6 \%$ das capivaras analisadas, porém o tronco braquiocefálico deu origem, primeiro à artéria subclávia esquerda, e em seguida trifurcou-se em artéria subclávia direita e artéria carótida comum direita e esquerda, diferindo completamente do encontrado no preá.

Uma terceira variação e, com menor frequência, identificada na formação do tronco braquiocefálico foi a origem das artérias carótidas comum direita e esquerda, por tronco comum, o tronco bicarotídeo. A presença do tronco bica- 
Quadro 1. Ramos colaterais do arco aórtico em mamíferos

\begin{tabular}{|c|c|}
\hline Espécie & Ramos colaterais \\
\hline Preá & $\begin{array}{l}\text { 1.Tronco braquiocefáfico: carótidas comum esquerda e direita e subclávia direita. } \\
\text { 2.Subclávia esquerda: vertebral, torácica interna, cervical superficial, o tronco costocervical e } \\
\text { artéria axilar. }\end{array}$ \\
\hline Bovinos e equinos & 1.Tronco braquiocefálico: subclávias direita e esquerda e tronco bicarotídeo \\
\hline Búfalo & $\begin{array}{l}\text { 1.Tronco braquiocefálico: subclávia esquerda, carótidas comuns direita e esquerda e subclávia } \\
\text { direita. }\end{array}$ \\
\hline Cão e gato & $\begin{array}{l}\text { 1.Tronco braquiocefálico: carótida comum direita e esquerda e subclávia direita. } \\
\text { 2.Subclávia esquerda: tronco costocervial, cervical profunda, vertebral, cervical superficial, to- } \\
\text { rácica interna e artéria axilar. }\end{array}$ \\
\hline Capivara & $\begin{array}{l}\text { 1.Tronco braquiocefálico: subclávia esquerda, carótida comum esquerda e tronco braquiocaro- } \\
\text { tídeo (carótida comum direita e subclávia direita) }\end{array}$ \\
\hline Chinchila & $\begin{array}{l}\text { 1.Tronco braquiocefálico: carótida comum esquerda, carótida comum direita e subclávia di- } \\
\text { reita. } \\
\text { 2. Subclávia esquerda }\end{array}$ \\
\hline Coelho & $\begin{array}{l}\text { 1.Tronco braquiocefálico: carótida comum esquerda e um pequeno tronco comum a carótida } \\
\text { direita e sublcávia direita. } \\
\text { 2. Subclávia esquerda }\end{array}$ \\
\hline Cutia & $\begin{array}{l}\text { 1.Tronco braquiocefálico: subclávia esquerda, carótida comum esquerda e um tronco comum a } \\
\text { carótida comum direita e subclávia direita. }\end{array}$ \\
\hline Lebre & $\begin{array}{l}\text { 1.Tronco braquiocefálico: tronco bicarotídeo e subclávia direita. } \\
\text { 2.Subclávia esquerda: torácica interna, intercostal dorsal, o tronco costocervical e as artéria ver- } \\
\text { tebral, cervical superficial e axilar }\end{array}$ \\
\hline Mocó & $\begin{array}{l}\text { 1.Tronco braquiocefálico: carótidas comum direita e esquerda e subclávia direita. } \\
\text { 2.Subclávia esquerda: vertebral, torácica interna, tronco costocervical e artérias cervical super- } \\
\text { ficial e axilar. }\end{array}$ \\
\hline Nutria & $\begin{array}{l}\text { 1.Tronco braquiocefálico: carótidas comum esquerda e direita e subclávia direita. } \\
\text { 2.Subclávia esquerda: vertebral torácica, torácica interna, vertebral e tronco comum cervical } \\
\text { superficial - profunda - escapular dorsal e axilar. }\end{array}$ \\
\hline Paca & $\begin{array}{l}\text { 1.Subclávia esquerda: vertebral, tronco costocervical, cervical superficial, axilar e torácica in- } \\
\text { terna. } \\
\text { 2.Tronco braquiocefálico: carótida comum esquerda e um tronco comum a cartódia direita e } \\
\text { subclávia direita. }\end{array}$ \\
\hline Porquinho-da-índia & $\begin{array}{l}\text { 1.Tronco braquiocefálico: carótida comum esquerda, carótida comum direita e subclávia direita } \\
\text { 2.Subclávia esquerda: tronco vertebral - escapular dorsal, tronco costocervical, cervical super- } \\
\text { ficial, axilar e torácica interna. }\end{array}$ \\
\hline Suíno & $\begin{array}{l}\text { 1.Tronco braquiocefálico: subclávia direita e tronco bicarotídeo } \\
\text { 2. Subclávia esquerda }\end{array}$ \\
\hline Tamanduá-mirim & $\begin{array}{l}\text { 1.Tronco braquiocefálico: subclávia direita e tronco bicarotídeo. } \\
\text { 2.Subclávia esquerda: vertebral, torácica interna, tronco costocervical e artéria cervical super- } \\
\text { ficial. }\end{array}$ \\
\hline Veado catingueiro & $\begin{array}{l}\text { 1.Tronco braquiocefálico: subclávia esquerda; carótida comum esquerda; subclávia direita; ca- } \\
\text { rótida comum direita }\end{array}$ \\
\hline Rato & 1.Tronco braquiocefálico: carótidas comum direita e esquerda e subclávias direita e esquerda. \\
\hline
\end{tabular}

Referência

Dyce et al. 2004

Cortellini et al. 2000

Kent 1983,

König \& Liebich 2011

Culau et al. 2007

Araújo et al. 2004

Sousa et al. 2013

Carvalho et al. 1993

Brudnicki et al. 2007.

Magalhães et al. 2007

Campos et al. 2010

Oliveira et al. 2001

Kabak \& Haziroglu 2003

König \& Liebich 2011

Pinheiro et al. 2011

Schimming et al. 2012

Hebel \& Stromberg 1986 rotídeo também foi relatado por Reckziegel et al. (2003) em $53,57 \%$ dos gambás dissecados, do qual 39,28\% surgiram do tronco braquiocefálico e em $14,28 \%$ o tronco bicarotídeo surgiu diretamente do arco aórtico, diferindo do preá. Já Magalhães et al. (2007) em 65\% dos mocós estudados, o tronco braquiocefálico originou primeiro a artéria subclávia direita e em seguida o tronco bicarotídeo, semelhante ao verificado no roedor estudado.

Quanto aos vasos que são emitidos da subclávia esquerda e direita, no preá, foram verificados a artéria vertebral, a artéria torácica interna, a artéria cervical superficial, o tronco costocervical e a artéria axilar. Este arranjo também foi descrito na paca (Oliveira et al. 2001), no tamanduá-mirim (Pinheiro et al. 2012) e na jaguatirica (Martins et al. 2010).

Na chinchila (Araújo et al. 2004), a artéria subclávia originou as artérias vertebral, escapular dorsal, tronco comum torácica interna-vertebral torácica e tronco comum as artérias cervical superficial - profunda. Na nutria (Cam- pos et al. 2010) a subclávia esquerda emite as artérias vertebral torácica, torácica interna, vertebral e tronco comum cervical superficial - profunda - escapular dorsal, continuando para o membro torácico esquerdo como artéria axilar. O comportamento da ramificação da artéria subclávia do preá difere do apresentado na chinchila e na nutria, haja visto que no preá não foi observado a formação do tronco comum as artérias cervical-superficial-profunda e o tronco comum cervical-superficial-profunda-escapular dorsal, observados na chinchila e na nutria, respectivamente. Resultados diferentes do encontrado para o preá também foram verificados por Santos et al. (2004), no mão-pelada quando relatam que a artéria vertebral não é ramo da artéria subclávia nesta espécie.

Em estudos realizados por Magalhães et al. (2007) no mocó, as artérias subclávia esquerda e direita ao longo do trajeto emitiram como ramos as artérias vertebral, torácica interna, tronco costocervical e artéria cervical superficial, continuando-se ao alcançar o espaço axilar, como artéria 
axilar, apresentando um maior calibre comparado aos demais ramos originados a partir da subclávia, semelhante ao verificado no preá.

Brudnicki et al. (2007) estudando o arco aórtico da lebre européia, relatam que a subclávia emite as artérias torácica interna, intercostal dorsal, o tronco costocervical e as artérias vertebral, cervical superficial e axilar. No preá a artéria intercostal dorsal não fez parte da ramificação da artéria subclávia.

\section{CONCLUSÕES}

0 arco aórtico do preá (Galea spixii Wagler, 1831) apresentou comportamento semelhante ao encontrado no porquinho-da-índia e na chinchila, sendo emitido dois ramos colaterais principais, o tronco braquiocefáfico e a artéria subclávia esquerda, acreditando-se que este seja o padrão para a espécie.

0 tronco braquiocefálico origina três artérias, representadas pela artéria carótida comum esquerda, a artéria carótida comum direita e a artéria subclávia direita, que podem emergir de pontos distintos ao longo do referido tronco.

As artérias subclávias direita e esquerda originam cinco vasos representados pela a artéria vertebral, a artéria cervical superficial, a artéria torácica interna, o tronco costocervical e a artéria axilar.

\section{REFERÊNCIAS}

Albuquerque J.F.G., Fonseca M.A.G., Carregal R.D., Souza W.M \& Bastos C.M.C. 1987. Contribuição ao estudo dos colaterais calibrosos do arco aórtico no coelho (Oryctolagus cuniculus Linnaeus, 1758). Ars Vet. 3(1):1-4.

Araújo A.C.P., Oliveira J.C.D \& Campos R. 2004. Ramos colaterais do arco aórtico e suas principais ramificações em chinchila (Chinchilla lanigera). Revta Port. Ciênc. Vet. 99:53-58.

Brudnicki W., Macherzyńska A. \& Nowicki W. 2007. Variation in the arteries of the aortic arch in european brown hare (Lepus europaeus). Electr. J. Pol. Agric. Univ. 10(1):1-5. Disponível em <http://www.ejpau.media. pl/volume10/issue1/art-03.html> Acesso em 15 ago. 2013.

Campos R., Araújo A.C.P. \& Azambuja R.C. 2010. Ramos colaterais do arco aórtico e suas principais ramificações em nutria (Myocastor coypus). Acta Sci. Vet. 38:139-146.

Carvalho M.A.M., Zanco N.A., Arrivabene M. \& Cavalcante Filho M.F. 1993. Ramos do arco aórtico na cutia (Dasyprocta aguti Rodentia). Anais XVI Congresso Brasileiro de Anatomia, São Paulo, p.122.

Cortellini L.M.F., Machado M.R.F., Oliveira F.S., Miglino M.A. \& Artoni S.M.B. 2000. Ramos do arco aórtico de bubalinos. Ciência Rural 30(3):445-448.

Culau P.O.V., Reckziegel S.H., Lindemann T., Araújo A.C.P. \& Balzaretti F. 2007. Colaterais do arco aórtico da capivara (Hydrochoerus hydrochaeris). Acta Scient. Vet. 35:89-92.

Domeniconi R.F., Abreu M.A.F., Benetti E.J. \& Villaça J.S. 2004. The contribution of the aortic branches in the vascularization of cervical regions, during the development of the nine banded armadillo (Dasypus novemcinctus Linnaeus, 1758). Int. J. Morphol. 22(2):113-118.
Dyce K.M., Sack W.O \& Wensing C.J. 2004. Tratado de Anatomia Veterinária. 3aㅡ ed. Elsevier, Rio de Janeiro. 813p.

Engel S., Maiochi R.R., Birck A.J., Filadelpho A.L. \& Guimarães G.C. 2013. Origem e distribuição do arco aórtico do cachorro-do-mato (Cerdocyon thous). Revta Cient. Eletr. Med. Vet. 11(21).

Fernandes Filho A. \& Borelli V. 1970. Contribuição ao estudo dos colaterais calibrosos do arco aórtico no gato. Revta Fac. Med. Vet. São Paulo 8(2):385-388.

Getty R. 1981. Anatomia dos Animais Domésticos. Vol.1. 5ae ed. Interamericana, Rio de Janeiro. 1134p.

Goshal N.G. 1981. Coração e artérias do carnívoro, p.1500-1513. In: Ibid. (Ed.), Anatomia dos Animais Domésticos. Vol.2. 5a ed. Interamericana, Rio de Janeiro.

Hebel R. \& Stromberg M.V. 1986. Anatomy and Embriology of the Laboratory Rat. BioMed Verlag, Wörthersee. 270p.

Kabak M. \& Haziroglu R.M. 2003. Subgross investigation of vessels originating from Arcus aortae in Guinea-pig (Cavia Porcellus). Anat. Histol. Embryol. 32:362-366.

Kent G.C. 1983. Comparative Anatomy of the Vertebrates. 5th ed. Mosby, St Louis, p.380-382.

König H.E. \& Liebich H.G. 2011. Anatomia dos Animais Domésticos: texto e atlas colorido. Artmed, Porto Alegre. 788p.

Magalhães M., Albuquerque J.F.G., Oliveira M.F., Papa P.C. \& Moura C.E.B. 2007. Ramos do arco aórtico no mocó (Kerodon rupestris). Revta Port. Ciênc. Vet. 102:49-52.

Martins D.M., Lima A.R., Pinheiro L.P., Brígida S.S.S., Araújo E.B., Melul R., Lacreta Júnior A.C.C., Menezes A.M.C., Souza A.C.B., Pereira L.C., Fioretto E.T. \& Branco E. 2010. Descrição morfológica dos ramos colaterais do arco aórtico e suas principais ramificações em Leopardus pardalis. Acta Vet. Brasilica 4(2):74-77.

Mendes B.V. 1987. Plantas e Animais para o Nordeste. Editora Globo, Rio de Janeiro. 167p.

Oliveira F.S., Machado M.R.F., Miglino M.A. \& Nogueira T.M. 2001. Gross anatomical study of the aortic ARC branches of the paca (Agouti paca Linnaeus, 1766). Braz. J. Vet. Res. Anim. Sci. 38:103-105.

Pinheiro V.L.C., Lima A.R., Pereira L.C., Gomes B.D. \& Branco E. 2012. Descrição anatômica dos ramos colaterais do arco aórtico do tamanduá-mirim (Tamandua tetradactyla). Biotemas 25(2):133-137.

Reckziegel S.H., Lindeman T. \& Culau P.O.V. 2003. Colaterais do arco aórtico no gambá (Didelphis albiventris). Ciência Rural 33(3):507-511.

Santos A.L.Q., Morais F.M., Malta T.S., Carvalho S.F.M. \& Alves Junior J.R.F. 2004. Topografia dos colaterais calibrosos do arco aórtico de um mão-pelada (Procyon cancrivoros Gray, 1865) (Carnivora - Procyoniadae) [The topography of the think collaterais of the aortic arch in a crab-eating raccoon Procyon cancrivorus Gray, 1865, Carnivora - Procyoniadae.] Archs Vet. Sci. 9(2):67-72.

Santos P.R.S., Carrara T.V.B., Silva L.C.S., Silva A.R., Oliveira M.F. \& Assis Neto A.C. 2011. Morphological characterization and frenquency of stages of the seminiferous epithelium cycle in captive bred Spix's Yellow-Toothed (Galea spixii Wagler, 1831). Pesq. Vet. Bras. 31(1):18-24.

Schimming B.C., Matteis R., Silva J.R.C.P. \& Guazzelli Filho J. 2012. Ramos do arco aórtico no veado-catingueiro. Revta Cient. Eletr. Med. Vet. 10(19).

Souza F., Bavaresco A.Z. \& Campos R. 2013. Ramos colaterais do arco aórtico e suas principais ramificações em coelho da raça Nova Zelândia (Oryctolagus cuniculus). Ciência Rural 43(12):2261-2267. 